Rev. Biol. Trop., 48(2/3): 313-316, 2000

www.ucr.ac.cr www.ots.ac.cr www.ots.duke.edu

\title{
Biomasa y densidad de dos especies de pastos marinos en ell sur de Quintana Roo, México
}

\author{
Martha Angélica Gutiérrez-Aguirre, María Gabriela de la Fuente-Betancourt y Adrián Cervantes- \\ Martínez \\ El Colegio de la Frontera Sur, Unidad Chetumal. Depto. de Pesquerías. A.P. 424, Chetumal, C.P. 77000. QROO, \\ México. Fax: (+983) 204-47, correo electrónico: martha@ecosur-qroo.mx.
}

Recibido 20-IV-1999. Corregido 18-II-2000. Aceptado 8-III-2000.

\begin{abstract}
The biomass and productivity of a seagrass community are useful for determining the ecological status of the coast. Leaf biomass and shoot density in beds of Thalassia testudinum Banks \& Sol. ex K. D. Koenig, were compared for two environments in the Mexican Caribbean coast ( $\mathrm{N}=6$ quadrants/site) in November 1998. Shoot and leaf biomass values were lower in the mangrove-associated meadow than in the reef lagoon meadow. This could be related to the higher percentage of epiphytes on the leaves. In addition, T. testudinum had more biomass than Syringodium filiforme Kütz in the reef lagoon.
\end{abstract}

Key words: Biomass, Caribbean, density, Mexico, seagrass.

Las praderas de pastos en la zona costera realizan tres funciones básicas: constituyen una fuente de alimentación para vertebrados, influyen en la biomasa relativa de una gran cantidad de organismos y estabilizan los sedimentos (Stoner 1980, Thorhaug 1986, van Tussenbroek 1995). En el Caribe mexicano existen tres especies de pastos: Halodule wrightii Aschers, Thalassia testudinum Banks \& Sol. ex K. D. Koenig y Syringodium filifor$m e$ Kütz; las dos últimas se consideran entre las plantas más productivas del mundo (Patriquin 1973, Gallegos et al. 1994). Desafortunadamente y a pesar de su importancia, los estudios en el Caribe dedicados a determinar la dinámica en la densidad, biomasa y productividad de los pastos marinos son escasos (van Tussenbroek 1995). El conocimiento de dichos factores permite disponer de información base, que habla del estado ecológico de los estuarios y de las zonas costeras y habili- ta detectar cambios o daños subsecuentes en la comunidad por medio del monitoreo (Dawes 1998). Es por ello que en el presente trabajo se elabora un estudio de la biomasa y densidad de T. testudinum y $S$. filiforme en el sur de Quintana Roo, México.

\section{MATERIALES Y MÉTODOS}

El trabajo de campo se realizó el 9 y 10 de noviembre de 1998, en dos sitios al sur de Quintana Roo: 1) la laguna arrecifal de Majagual y 2) frente a un manglar de Boca Bacalar Chico, ambos con alrededor de $1 \mathrm{~m}$ de profundidad. Majagual (M) es una playa arenosa con praderas de T. testudinum y $S$. filiforme, ubicada a $18^{\circ} 43^{\prime} \mathrm{N}$ y $87^{\circ} 42^{\prime} \mathrm{W}$. En contraste, la costa de Boca Bacalar Chico (BB) está dominada por los manglares Rhizophora mangle $\mathrm{L}$. y Conocarpus erecta L.; es un sitio con bajas 
corrientes, sedimentos abundantes de fácil suspensión y una pradera de $T$. testudinum, ubicada a $18^{\circ} 10^{\prime} \mathrm{N}$ y $87^{\circ} 38^{\prime} \mathrm{W}$.

En cada sitio se colocaron aleatoriamente seis cuadrantes de $20 \times 20 \mathrm{~cm}$. Se cortaron las vainas de pastos a nivel del sustrato dentro del cuadrante y se determinó la cantidad de hojas por vaina y el número de vainas por cuadrante. Con el material obtenido se determinó la biomasa foliar en peso seco y húmedo de cada especie. En T. testudinum se midió la longitud del tejido fotosintético (verde), muerto y ancho foliar. Visualmente se determinó el porcentaje de epífitas y se calculó la edad promedio en días de 14 a 16 vainas, considerando el número de cicatrices/vaina (Patriquin 1973). En S. filiforme se determinó la longitud total de la hoja. Adicionalmente, se tomaron tres muestras de sedimento por sitio. Cada muestra se dividió en tres réplicas para medir el porcentaje prome- dio de materia orgánica (por determinación de cenizas) y el tamaño medio de grano de cada lugar (Folk 1969).

\section{RESULTADOS}

Al comparar las características de T. testudinum en los dos sitios de muestreo (Cuadro 1), se encontraron diferencias significativas en la longitud del tejido fotosintético (Student $\mathrm{p}<0.05$, $\mathrm{t}=3.14$ ), muerto, porcentaje de epífitas sobre las hojas (Student $\mathrm{p}<0.05, \mathrm{t}=3.71 \mathrm{en}$ ambos) y edad de las vainas (Student $\mathrm{p}<0.05, \mathrm{t}=2.12$ ). $\mathrm{El}$ ancho de las hojas fue similar en ambos sitios (Student $\mathrm{p}>0.05, \mathrm{t}=-0.171)$. Así mismo, se observó que el número promedio de vainas con dos hojas fue mayor, mientras que el número promedio de hojas por vaina fue similar; en general, la edad promedio de las vainas de T. testudinum no excedió los 520 días (Cuadro 1).

\section{CUADRO 1}

Producción de hojas, vainas y epífitas de Thalassia testudinum y Syringodium filiforme en Majagual y Boca Bacalar Chico, Quintana Roo, México (área $=0.04 \mathrm{~m} 2$ )*.

\begin{tabular}{|c|c|c|c|c|c|c|}
\hline & Majagual & & Majagual & & B. Bacalar Chico & \\
\hline & T. testudinum & $\mathrm{N}$ & S. filiforme & $\mathrm{N}$ & T. testudinum & $\mathrm{N}$ \\
\hline Vainas con 1 hoja & $3.5 \pm 2.12$ & 2 & $30.33 \pm 12.85$ & 3 & $2 \pm 1$ & 3 \\
\hline Vainas con 2 hojas & $20.33 \pm 5.50$ & 3 & $25.66 \pm 11.37$ & 3 & $8.75 \pm 4.27$ & 4 \\
\hline Vainas con 3 hojas & $15.66 \pm 2.08$ & 3 & & & $4.5 \pm 4.50$ & 4 \\
\hline Vainas con 4 hojas & $1 \pm 0.0$ & 2 & & & $1.5 \pm 0.70$ & 2 \\
\hline Vainas con 5 hojas & 1 & 1 & & & & \\
\hline Vainas/cuadrante & $31.83 \pm 11.33$ & 6 & $46.33 \pm 18.64$ & 6 & $15.33 \pm 5.50$ & 6 \\
\hline Total de vainas & 120 & 3 & 168 & 3 & 62 & 4 \\
\hline Total de hojas & 283 & 3 & 245 & 3 & 142 & 4 \\
\hline $\begin{array}{l}\text { Promedio de hojas } \\
\text { por vaina }\end{array}$ & 2.35 & 3 & 1.4 & 3 & 2.29 & 4 \\
\hline Peso húmedo (g) & $54.66 \pm 20.20$ & 3 & $9.66 \pm 4.50$ & 3 & $28.25 \pm 13.86$ & 4 \\
\hline Peso seco (g) & $11.66 \pm 1.15$ & 3 & $1.66 \pm 0.57$ & 3 & $5.50 \pm 2.64$ & 4 \\
\hline L. tejido verde $(\mathrm{cm})$ & $28.68 \pm 2.81$ & 30 & $31.86 \pm 4.32$ & 30 & $33.14 \pm 7.19$ & 30 \\
\hline L. tejido muerto $(\mathrm{cm})$ & $1.50 \pm 1.85$ & 30 & & & $5.04 \pm 5.40$ & 30 \\
\hline Ancho (mm) & $11.80 \pm 2.26$ & 30 & & & $11.46 \pm 1.30$ & 30 \\
\hline Epífitas (\%) & $14.6 \pm 9.42$ & 30 & & & $28.59 \pm 18.11$ & 27 \\
\hline Edad (días) & $302.57 \pm 139.31$ & 14 & & & $512.43 \pm 347.31$ & 16 \\
\hline
\end{tabular}

*La edad se representa para T. testudinum. El n<10 representa al número de cuadrantes, el n $>10$ representa al número de hojas, vainas y rizomas. 
El número de vainas/cuadrante de T. testudinum, así como la biomasa foliar en peso seco y húmedo, fueron significativamente más elevados en $\mathrm{M}$ que en BB ( $U$ MannWhitney $\mathrm{p}=0.028$ y 0.05 respectivamente). No se observaron diferencias significativas entre el número de vainas/cuadrante de T. testudinum y $S$ filiforme de M ( $U$ Mann-Whitney $\mathrm{p}=0.155$ ), pero la biomasa en peso seco y húmedo de la primera, fue significativamente mayor ( $U$ Mann-Whitney $\mathrm{p}=0.05$ ). Por otro lado, el porcentaje promedio de materia orgánica fue significativamente más elevado en los sedimentos de BB $(6.1 \%, \mathrm{n}=3)$, que en los sedimentos de M $(4.0 \%, \mathrm{n}=4)$ ( $U$ MannWhitney $\mathrm{p}=0.01)$. El tamaño medio de partícula encontrado, correspondió al de arena media para ambos sitios.

\section{DISCUSIÓN}

Los resultados muestran que la biomasa de $T$. testudinum fue mayor que la de $S$. filifor$m e$, contrario a lo obtenido por Gallegos et al. (1994) y van Tussenbroek (1995) en estudios realizados al norte de Quintana Roo (extrapolando los datos por $\mathrm{m}^{2}$ ). Probablemente el efecto de fuertes corrientes como resultado de la influencia del huracán Mitch durante la primer semana de noviembre de 1998 , previo a la fecha de colecta, produjo mayor pérdida de tejido foliar de $S$. filiforme. Por otro lado, aunque el número de vainas fue variable para ambas especies, los resultados son similares a lo observado por Patriquin (1973) en Bermudas y Barbados para T. testudinum.

La pradera de T. testudidum de BB se encuentra frente a un manglar en una zona protegida de baja energía. Aunque tales condiciones provocan biomasa y productividad elevadas en la especie (Patriquin 1973, van Tussenbroek 1995), la pradera frente al manglar presentó menor biomasa y menor número de vainas por cuadrante, que la pradera establecida en la laguna arrecifal. Un factor que puede explicar lo anterior es la mayor cantidad de epífitas sobre las hojas de $T$. testudinum de BB, ya que se reconoce que éstas pueden tener efectos deletéreos sobre la biomasa. Adicionalmente, las vainas de BB fueron más viejas que las de $\mathrm{M}$, lo cual pudo disminuir la cantidad de hojas generadas por vaina y en consecuencia, la biomasa (Patriquin 1973). La altura de las hojas fue mayor en $\mathrm{BB}$, lo mismo que el contenido de materia orgánica en los sedimentos del sitio por la influencia del manglar próximo a la pradera de T. testudinum.

Los resultados aquí expuestos pueden funcionar como base para estudios subsecuentes, ya que representan una visión preliminar de las comunidades de pastos marinos del sur de Quintana Roo, sobre todo considerando que dichas comunidades muestran variaciones naturales (significativas) de crecimiento, biomasa y producción primaria dentro y entre las mismas a través del tiempo (van Tussenbroek 1995). Es importante considerar otros factores que pueden influir en la dinámica de los pastos, por ejemplo, el efecto de los herbívoros, la tasa de penetración de luz y el efecto de las corrientes.

\section{AGRADECIMIENTOS}

El estudio es resultado del trabajo realizado durante el curso "Vegetación costera tropical", bajo la dirección de Julio Espinoza Avalos dentro del programa de posgrado del Colegio de la Frontera Sur (ECOSUR), unidad Chetumal, México apoyado por el Consejo Nacional de Ciencia y Tecnología (CONACYT). Los comentarios de tres revisores anónimos, mejoraron el contenido del estudio.

\section{RESUMEN}

La biomasa y productividad de una comunidad de pastos marinos es útil para determinar el estado ecológico de la costa. Se comparó la biomasa foliar y la densidad de vainas en praderas de Thalassia testudinum Banks \& Sol. ex K. D. Koenig, en dos ambientes de la costa del Caribe mexicano $(\mathrm{N}=6$ cuadrantes/sitio) en Noviembre de 1998. Los valores de vainas y biomasa foliar fueron más bajos en la pradera asociada al manglar, que en la pradera establecida en una laguna arrecifal. Esto probablemente se deba a que las hojas frente al manglar tuvieron porcentaje más alto de epífitas. Por otro lado, T. testudinum tuvo mayor biomasa que Syringodium filiforme Kütz en la laguna arrecifal. 


\section{REFERENCIAS}

Dawes, C. J. 1998. Biomass and photosynthetic responses to irradiance by a shallow and a deep water population of Thalassia testudinum on the west coast of Florida. Bull. Mar. Sci. 62: 89-96.

Folk, R.L. 1969. Petrología de las rocas sedimentarias. Traducción del inglés por Schlaepfer, C. y R. M. de Schmitter. Instituto de Geología de la Universidad Nacional Autónoma de México, México D. F., México. 405 p.

Gallegos, M. E., M. Merino, A. Rodríguez, N. Marbà \& C. M. Duarte. 1994. Growth patterns and demography of pioneer Caribbean seagrasses Halodule wrightii and Syringodium filiforme. Mar. Ecol. Prog. Ser. 109: 99-104.
Patriquin, D. 1973. Estimation of growth rate, production and age of the marine angiosperm Thalassia testudinum König. Carib. J. Sci. 13: 111-123.

Stoner, A. W. 1980. The role of the seagrass biomass in the organization of benthic macrofaunal assemblages. Bull. Mar. Sci. 30: 537-551.

Thorhaug, A. 1986. Review of seagrass restoration efforts. Ambio 15: 110-117.

van Tussenbroek, B. I. 1995. Thalassia testudinum leaf dynamics in a Mexican Caribbean coral reef lagoon. Mar. Biol. 122: 33-40. 